\title{
The effect of the improperly scanned scan body images on the accuracy of virtual implant positioning in computer-aided design software
}

\author{
Se-Won Park ${ }^{1}$, Yong-Do Choi ${ }^{1}$, Du-Hyeong Lee ${ }^{1,2 *}$ \\ 'Department of Prosthodontics, School of Dentistry, Kyungpook National University, Daegu, Republic of Korea \\ ${ }^{2}$ Institute for Translational Research in Dentistry, Kyungpook National University, Daegu, Republic of Korea
}

\begin{abstract}
PURPOSE. The aim of this study was to examine the importance of the defect-free scanning of a scan body by assessing the accuracy of virtual implant positioning in computer-aided design (CAD) software when the scan body image is improperly scanned. MATERIALS AND METHODS. A scan body was digitized in a dentiform model using an intraoral scanner, and scanned images with differing levels of image deficiency were generated: $5 \%$, $10 \%$, and $15 \%$ deficiency in the flat or rounded area. Using a best-fit image matching algorithm on each of the deficient scan body images, corresponding virtual implants were created. The accuracy of the implant position was evaluated by comparing the linear and angular discrepancies between the actual and virtual positions of the implant. Kruskal-Wallis tests and Mann-Whitney $\mathrm{U}$ tests with Bonferroni correction were used to determine the statistical differences among the seven scanned image deficiency groups $(\alpha=.05)$. RESULTS. In general, the linear and angular discrepancies of the implant position in the software increased as the deficiency of the scan body images increased. A $15 \%$ scan body image deficiency generated larger discrepancies than deficiency of $5 \%$ and $10 \%$. The difference of scan defect position, flat or rounded area, did not affect the accuracy of virtual implant orientation at $5 \%$ and $10 \%$ deficiency level, but did affect the accuracy at $15 \%$ deficiency level. CONCLUSION. Deficiencies in the scanned images of a scan body can decrease the accuracy of the implant positioning in CAD software when the defect is large, thus leading to the incorrect fabrication of implant prostheses. [J Adv
\end{abstract}

Prosthodont 2020;12:107-13]

KEYWORDS: Scan body; Implant; Image deficiency; Accuracy; Image registration

\section{INTRODUCTION}

Conventional impression methods were first developed in the mid-18th century to model the three-dimensional (3D)

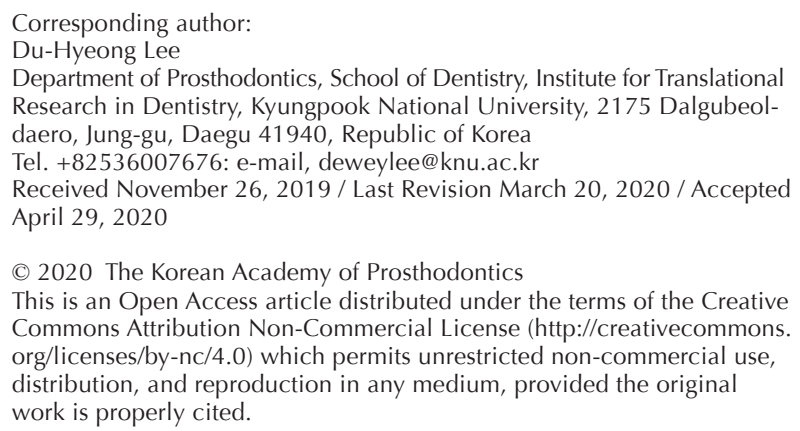

This work was supported by the National Research Foundation of Korea (NRF) grant funded by the Korea government (MSIP) (2017R1C1B2004976). geometry of the oral cavity. ${ }^{1,2}$ The complex workflow required for analog impressions, and the contact between the tray and the intraoral anatomic structures could create discomfort for both the patient and the clinician. ${ }^{3}$ In addition to being inconvenient, traditional alginate and silicone impressions have a number of other limitations, such as the lack of precise replication, poor dimensional stability, flaws in the finish lines of the abutment, limited working time, the possibility of cross infection, and allergic reactions to the impression material. ${ }^{4-6}$ Moreover, the dimensional expansion and distortion of the stone cast during setting can further reduce the accuracy of the conventional prosthesis fabrication process, resulting in an ill-fitting final prosthesis. $^{7}$

The introduction of computer-aided design and computer-aided manufacturing (CAD/CAM) to dental prosthodontics has led to conventional fabrication methods being gradually replaced with CAD/CAM approaches., ${ }^{2,8}$ Digital 
impressions have overcome many of the drawbacks of the analog impression-making process by eliminating the use of impression material and gypsum casts. ${ }^{9-11}$ Digital impression images can be created using indirect or direct digitization. ${ }^{12}$ In an indirect digitization workflow, the surface image is obtained by scanning a master cast or a silicone impression body using a laboratory scanner. ${ }^{12}$ In a full digitization workflow, the data acquisition process is conducted with an intraoral scanner. ${ }^{3}$ Intraoral digital scanning enables clinicians to directly acquire surface data from the oral cavity without a silicone impression or a stone cast. ${ }^{2}$

In the direct digital impression of implants, an intraoral scanner is employed in conjunction with an implant scan body. ${ }^{13}$ By attaching the scan body to the implant and scanning it, the 3D position of the implant in the bone can be acquired. ${ }^{14}$ The scanned image of the scan body is imported into dedicated CAD software, in which the scan body is recognized using an image superimposition algorithm that works off a design template for the corresponding scan body that is embedded in the software. ${ }^{14}$ Based on the 3D position of the virtual implant, customized abutments and final prostheses can be designed. ${ }^{2}$ The scan body can thus be considered a transfer medium that connects the actual position of the implant in the jaw with the position of the virtual implant in the CAD software. ${ }^{15}$ As such, the accurate digitization of the scan body in the oral cavity is essential for correctly registering the corresponding position of the virtual implant in the CAD program.

The surface image acquisition process for the scan body is important because the scan data provides the congruent area necessary for image matching. ${ }^{15}$ The digital scanning of a scan body via intraoral scanners has been reported to be as accurate as traditional physical impressions in in vitro study design. ${ }^{16}$ However, when intraoral scanning is employed in a patient's mouth, the quality of the scan body image can be compromised. ${ }^{10}$ Scanning requires a direct line of sight for light to the area of interest, ${ }^{17}$ something which may be restricted in clinical applications due to the poor positioning of the scanning wand in the oral cavity arising from the fact that wide mouth opening can be limited in some patients. ${ }^{18}$ The local anatomy of the oral cavity and the position of the scan body can also lead to the faulty acquisition of scan body images. ${ }^{17}$ In particular, the teeth adjacent to the scan body can obscure the view of its proximal surface, leading to incomplete scanned images.

Although several studies have investigated the accuracy and feasibility of intraoral digital scanning, ${ }^{10,12,13,16}$ the effect of improperly scanned data of a scan body on the accurate identification of the actual position of the implant has not yet been addressed. The purpose of this study was thus to evaluate the effects of this improper scan data on the accuracy of virtual implant positioning in CAD software. The null hypothesis was that the accuracy of virtual implant positioning is unaffected by the degree of deficiency in the surface scan data for the scan body.

\section{MATERIALS AND METHODS}

Figure 1 presents the workflow for this study. A dental implant (AnyOne, MegaGen, Daegu, Korea; $\varnothing 4.0 \times 10$ $\mathrm{mm}$ ) was installed in a dentiform model in the area of the left maxillary first molar. A cylindrical scan body (AnyOne

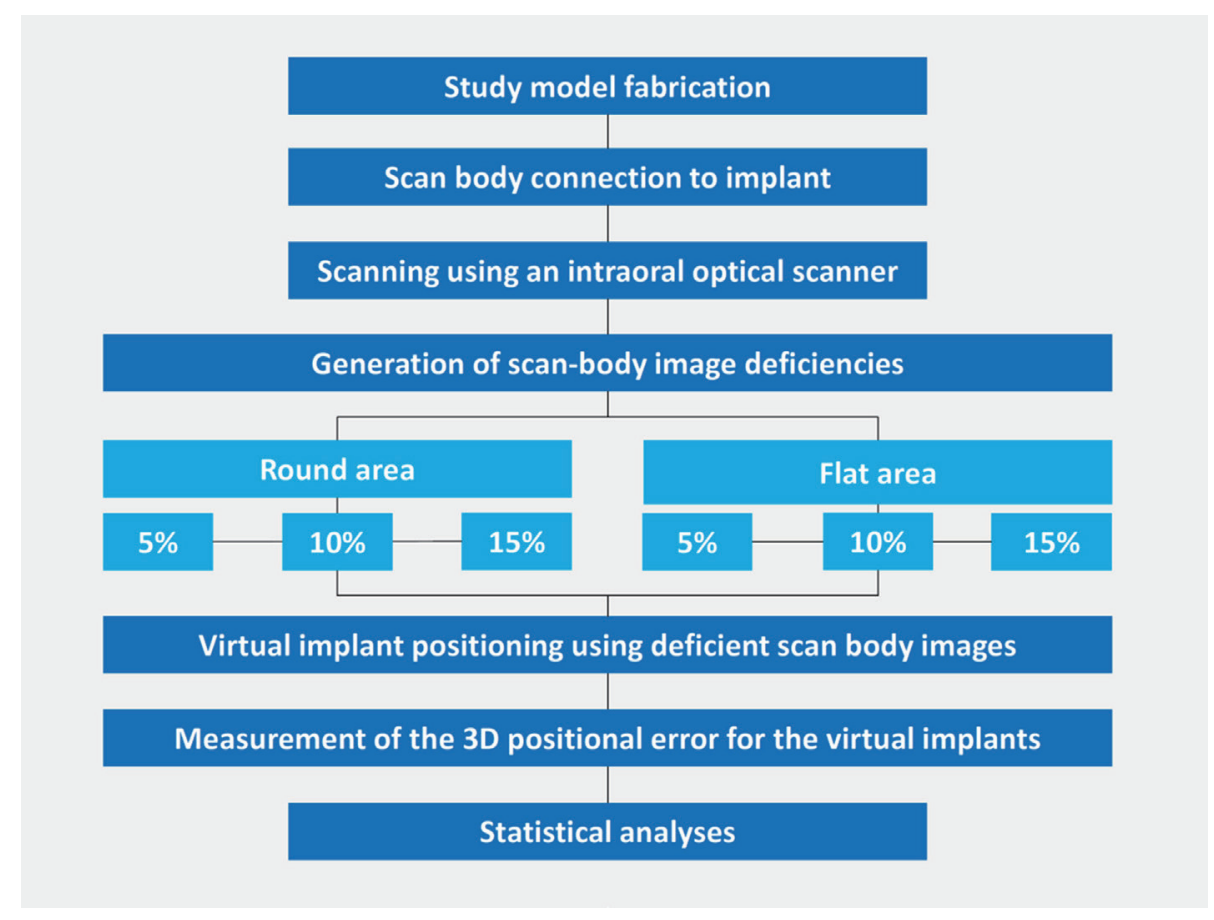

Fig. 1. Workflow of the present study. 
Scan abutment, MegaGen, Daegu, Korea; $\varnothing 4.0 \times 9 \mathrm{~mm})$ was connected to the implant with an abutment screw (Fig. 2). The scan body was fabricated from polyetheretherketone (PEEK) and contained both rounded and flat lateral sections. The scan body and surrounding gingiva were digitized using an intraoral optical scanner (CS3600, Carestream, Rochester, NY, USA). Smooth and uninterrupted surfaceimage acquisition was conducted while maintaining a distance of approximately $5 \mathrm{~mm}$ from the tip of the intraoral scanner to the scan body.

To generate the improperly scanned data around the scan body, the fully scanned image of the scan body was transformed using dental image software (Dental Imaging, Carestream, Rochester, NY, USA). A certain percentage of the surface of the scan body was removed $(5,10$, or $15 \%$ ) from either the flat or rounded section of the scan body using protractor software (Screen Protractor 4.0, Iconico, New York, NY, USA) (Fig. 3A, B). This means that there

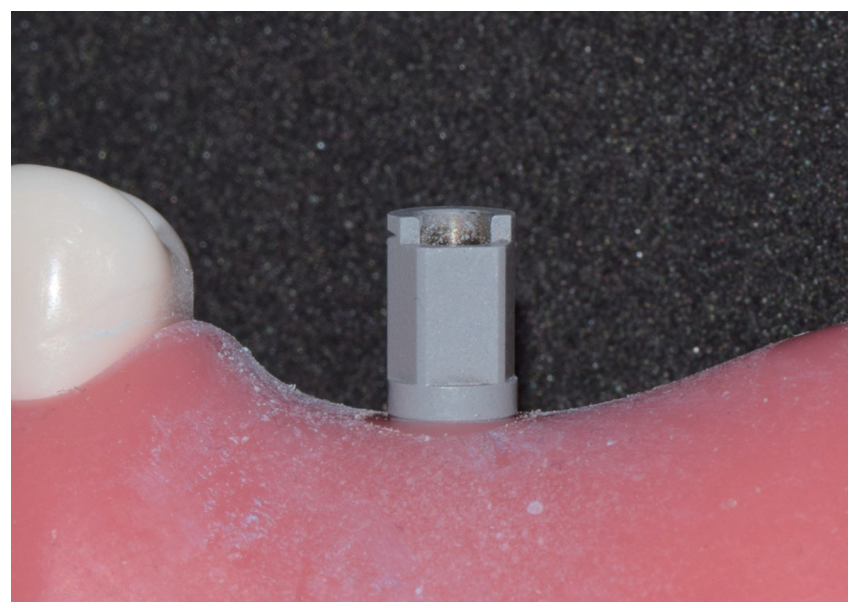

Fig. 2. Scan body connected to the implant in the dentiform model. were seven experimental conditions investigated in this study: no deficiency (i.e., the fully scanned image), $5 \%, 10 \%$, and $15 \%$ deficiency in the flat section, and 5\%,10\%, and $15 \%$ deficiency in the rounded section. After this surface modification process, automatic image correction on the deficient area was conducted using the same dental image software (Fig. 3C).

The scanned images were then imported into dental CAD software (IDC S1, Amann Girrbach, Koblah, Austria). The scan body images were superimposed onto the scan body design template embedded in the CAD software using a best-fit image registration algorithm. Consequently, a virtual implant was created for each experimental condition based on the matched scan body design (Fig. 4). The scan body and implant images were then extracted as a single standard tessellation language (STL) file (scan body-andimplant image). This process was repeated twelve times for each of the seven levels of scan deficiency, thus generating a total of 49 STL files for all groups combined. The sample size was determined with a power analysis to provide statistical significance $(\alpha=.05)$ at $80 \%$ power.

To analyze the $3 \mathrm{D}$ geometric accuracy of the virtual implant positioning, the scan body-and-implant images were superimposed onto a reference image using the implant portion of the image as the congruent area (Fig. 5). ${ }^{19}$ The reference image was produced in the CAD software to reflect the correct virtual implant position relative to the scan body template image. Accordingly, after the superimposition of the implant images, the error in the scan body image matching results was calculated by comparing the positional discrepancy between the scans and the design template using analysis software (Geomagic DesignX, 3D Systems, Morrisville, NC, USA; Fig. 6). The linear discrepancy was measured at the top of the scan body using the two center points of the test images and the reference image, while the angular discrepancy was assessed using the two centerlines of the test images and the reference image.
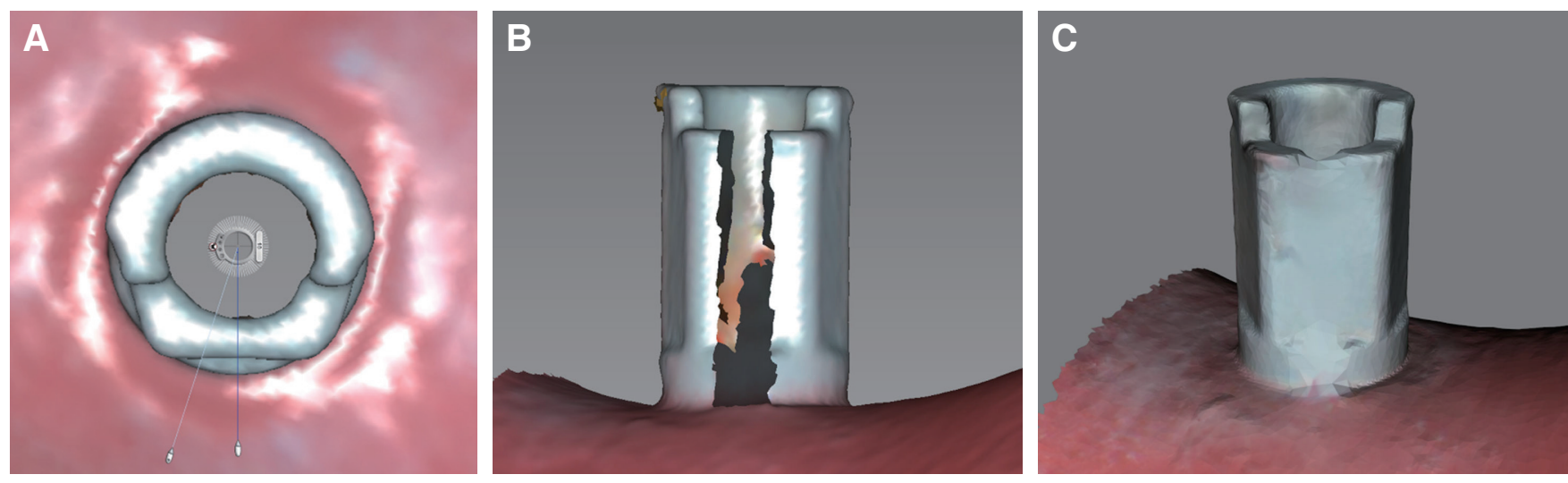

Fig. 3. (A) Illustration of the areas removed to generate the improperly scanned data around a scan body, (B) Scan body image with an improper scan data on the flat section, and (C) Scan body image after automatic image correction of the deficient area. 


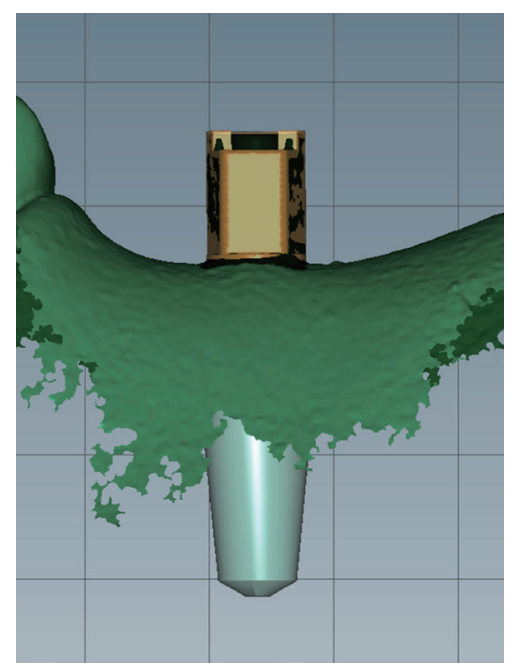

Fig. 4. Image matching of the acquired scan body image and the design template image for the scan body, and the creation of the virtual implant using CAD software.
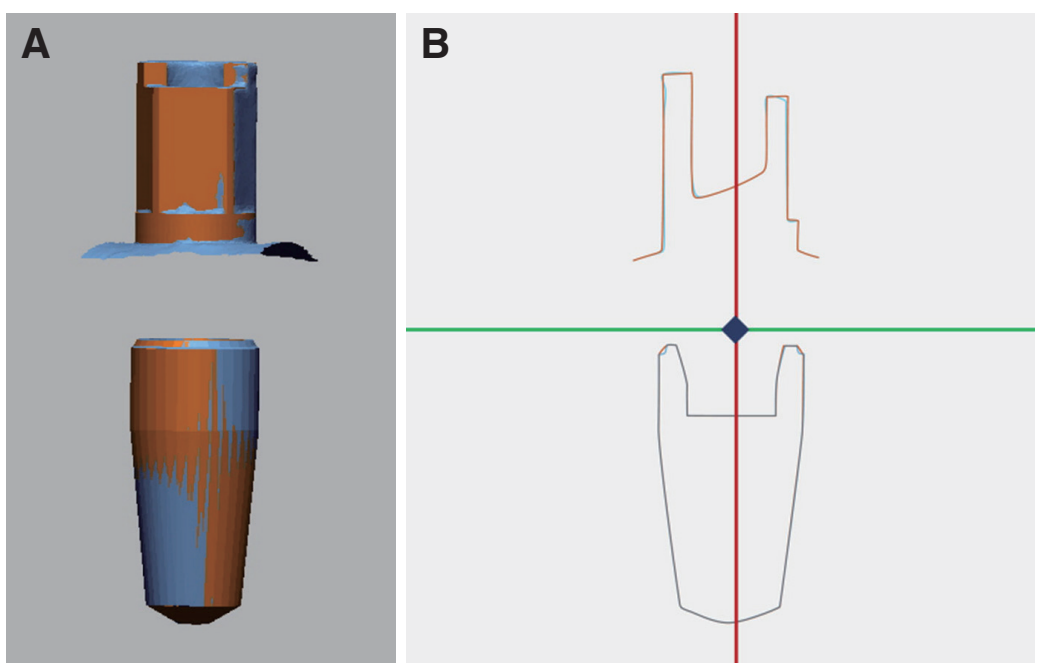

Fig. 5. Superimposition of the scan body-and-implant image onto the reference image based on the implant section. (A) 3D view, (B) Crosssectional view.
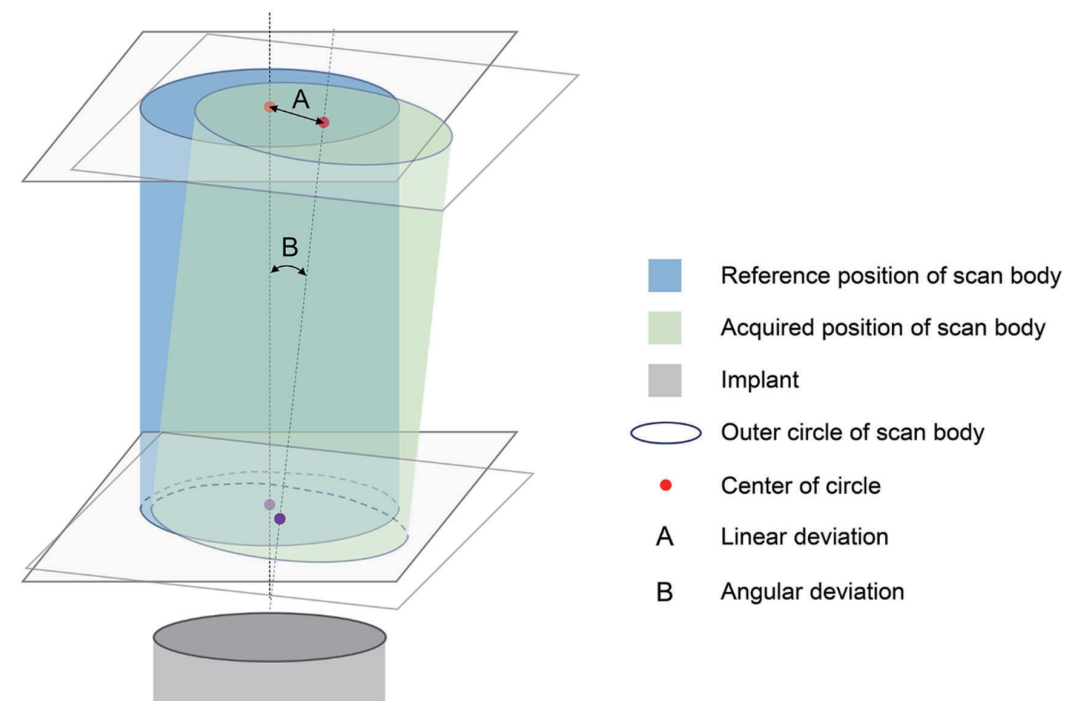

Fig. 6. Measurement of the 3D linear and angular positional discrepancies between the acquired and reference scan body images.

To detect the center points, a virtual plane perpendicular to the long axis of the scan body was set at the top level of the scan body image, and a circle line was then automatically expressed on the virtual plane by recognizing the cross-sectional image of the scan body. The center point was determined based on the circle line.

All discrepancy measurements were analyzed using statistical analysis software (IBM SPSS Statistics v23.0 for Windows, IBM Corp., Armonk, NY, USA) Kruskal-Wallis tests, with post-hoc Mann-Whitney $U$ tests and Bonferroni correction, were used to identify any statistical differences between the seven levels of scan body image deficiency in terms of the accuracy of virtual implant positioning. MannWhitney $\mathrm{U}$ tests were used to investigate the effect of where the image deficiency occurred (i.e., on the rounded or flat surface of the scan body) for the same image deficiency levels. Interactions between the level of image deficiency and the deficiency location on the implant positional discrepancy were statistically investigated with two-way analysis of variance (ANOVA). The significance level was set at 0.05 . 


\section{RESULTS}

Overall, the accuracy of the virtual implant positioning in the CAD software was influenced by both the level of scanimage deficiency and where the deficiency occurred. The linear discrepancy generally worsened as the deficiency level of the scan body image increased $(P<.05$; Table 1$)$, though there was no significant difference between the images with no deficiency and those with 5\% and 10\% deficiency. The angular discrepancy tended to increase with a rise in the level of scan body image deficiency $(P<.05)$; however, this was not statistically consistent (Table 2 ).

In terms of the effects of position of the scan body image deficiency occurred, defects in the flat and rounded sections of the scan body did not generate significantly different error values for $5 \%$ and $10 \%$ deficiency levels $(P=$ .143 at $5 \%$ deficiency, $P=.713$ at $10 \%$ deficiency) (Table 1 ). For a $15 \%$ deficiency level, the discrepancy was lower for the rounded section than for the flat section $(P<.001)$. There was no specific trend in the angular discrepancy with regards to where the deficiency occurred as the deficiency level increased.

Interactions between the level of image deficiency and the deficiency location on the implant positional discrepancy were statistically investigated with two-way analysis of variance (ANOVA). Two-way ANOVA showed no significant interaction between the level of image deficiency and the deficiency location on the implant positional discrepancy $(\mathrm{F}=2.059, P=.136)$.

\section{DISCUSSION}

The results of the present study revealed differences in the accuracy of virtual implant positioning in CAD software depending on the level of deficiency in the scanned images of the scan body. As the image deficiency level increased, the virtual implant positioning became generally more inaccurate. It was found that a scan body image deficiency of more than 15\% generated higher linear and angular discrepancies in the positioning of virtual implants than images with $5 \%$ or $10 \%$ image deficiency. Because significant differences in the accuracy of virtual implant positioning were found between the images with 15\% deficiency level, but not with below $10 \%$, the null hypothesis of the study was partially rejected. It was also confirmed that the location of the deficiency in the scan body image could affect the quality of image matching in CAD software when the defect is large. Thus, when intraoral scans are conducted in a clinic, the acquisition of sufficient surface data for the scan body is crucial for the accurate virtual modeling of the position of the actual implant in the oral cavity. Deficient images of the scan body could subsequently lead to ill-fitting implantsupported fixed prostheses in the marginal and proximal contact areas within the oral cavity.

In this study, the misalignment of the virtual implant arose when the scan body image was improper. The accuracy of virtual implant positioning was significantly lower for large image defects compared to minor image defects due to image registration errors for the scan body, which meant

Table 1. Linear discrepancy (mean $\pm S D ; \mu m$ ) in virtual implant positioning according to the scan body image deficiency level and deficiency location

Deficiency level (\%)

\begin{tabular}{lccccc} 
Deficiency location & 0 & 5 & 10 & 15 & $P$ \\
\hline Flat & $47.9 \pm 2.6^{\mathrm{a}}$ & $48.0 \pm 2.3^{\mathrm{a}}$ & $50.4 \pm 2.8^{\mathrm{a}}$ & $81.6 \pm 2.0^{\mathrm{b}}$ & $<.001$ \\
Round & $47.7 \pm 1.9^{\mathrm{a}}$ & $49.8 \pm 2.7^{\mathrm{a}}$ & $74.1 \pm 3.9^{\mathrm{b}}$ & $<.001$ \\
$P$ & & .143 & .713 & $<.001$ & \\
\hline
\end{tabular}

Significant differences within the same row are represented by different superscript letters.

Table 2. Angular discrepancy (mean \pm SD; degree) in virtual implant positioning according to the scan body image deficiency level and deficiency location

Deficiency level (\%)

\begin{tabular}{lccccc} 
Deficiency location & 0 & 5 & 10 & 15 & $P$ \\
\hline Flat & & $0.16 \pm 0.02^{\mathrm{a}}$ & $0.18 \pm 0.01^{\mathrm{b}}$ & $0.26 \pm 0.01^{\mathrm{c}}$ & $<.001$ \\
Round & $0.17 \pm 0.01^{\mathrm{a}}$ & $0.17 \pm 0.01^{\mathrm{a}}$ & $0.18 \pm 0.01^{\mathrm{a}}$ & $0.24 \pm 0.01^{\mathrm{b}}$ & $<.001$ \\
$P$ & & .052 & .160 & .001 & \\
\hline
\end{tabular}

Significant differences within the same row are represented by different superscript letters. 


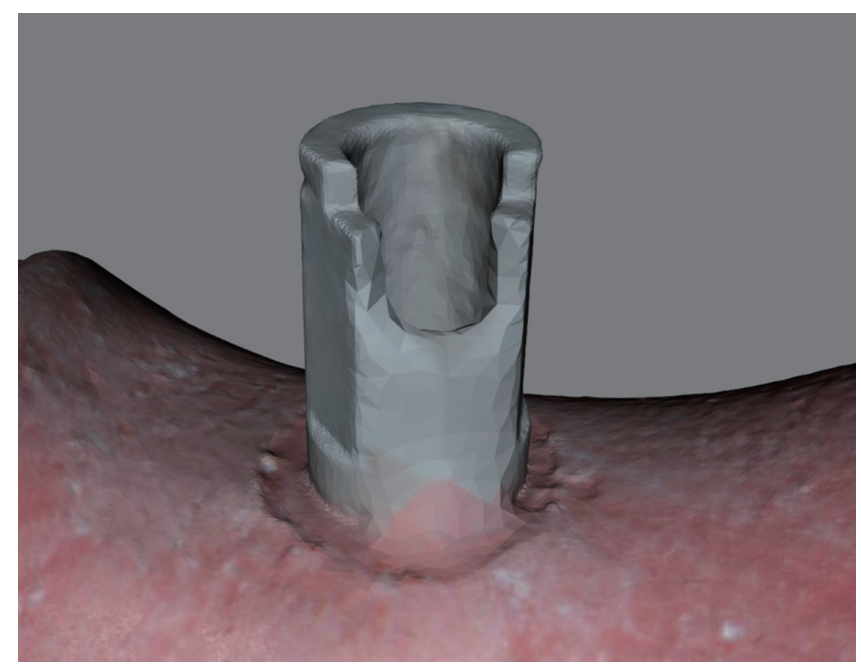

Fig. 7. Inaccurate stitching process of surface filling in the cases of large image defect.

accurate image matching was impossible. When conducting 3D scans, a scanner utilizes a variety of morphological characteristics from the target object as a reference when stitching the scan images together. ${ }^{20-22}$ When a particular area of the object is not scanned, the software in the scanner attempts to fill the empty space by bridging adjacent surfaces. ${ }^{17}$ This process is sufficient if the defect is small and the neighboring areas are morphologically similar. ${ }^{17}$ However, for large defects, difficulties may arise in the stitching process because of the lack of reference landmarks and the gradual accumulation of error; thus, large defects are likely to lead to inaccurate surface filling, which often manifests as artificial bulges or hollows. ${ }^{23}$ This failure in the stitching process was observed in the present study (Fig. 7). Image reconstruction software is also vital to the quality of a scanned image. ${ }^{24}$ Therefore, in addition to sufficient scan-image acquisition, the stitching algorithm may need to be improved to reduce the faulty processing of incomplete information.

The accuracy of intraoral digital impressions has been documented to be acceptable and comparable with that of traditional silicone impressions for partial fixed dental prostheses. ${ }^{16,25-27}$ The trueness of intraoral scanning reportedly ranges between $43.5 \mu \mathrm{m}$ and $137.0 \mu \mathrm{m}$ in various manufacturing systems. ${ }^{28}$ However, when a scanner is employed in a clinic, a number of clinical and operator issues can influence scan accuracy, leading to differences from the results of in vitro research. For example, the camera position during the intraoral scanning process clearly affects accuracy. ${ }^{17}$ In clinical practice, a smaller scanner head is more likely to maintain a direct line of sight for light during the entire scanning process for objects located in narrow spaces, particularly in the distal molar area. However, according to Hayama et al., ${ }^{29}$ the use of a larger scanner head leads to improved trueness and precision compared to a smaller head because a larger head requires fewer scanned images, which could increase accuracy. ${ }^{29}$ Thus, in intraoral scanning, it may be more effective to scan the general area using a larger head to increase the imaging area and then make additional scans of a target narrow area using a smaller head. The scan strategy is another major factor influencing the quality of a scan. ${ }^{30}$ Müller et al ${ }^{31}$ found significant differences in the accuracy of an intraoral scanner when the scan strategy was altered. Similarly, operator experience may also affect the scan results because greater experience is likely to be associated with wider knowledge of the available scan strategies. Indeed, Giménez et al. ${ }^{32}$ reported better scan results from experienced operators than from inexperienced operators.

This study is believed to be the first to investigate the 3D accuracy of virtual implant positioning in CAD software when the scan body attached to the implant is improperly scanned. The incomplete scanning of a scan body is likely to happen in clinical uses when the line of sight for light during the scanning process is restricted, and this study systematically demonstrated the adverse effects of scan body image defects on the accuracy of the position of the virtual implant. However, this study was limited in terms of its in vitro design and the lack of consideration of patientrelated restrictions. Intraoral scan data acquired from oral cavities may not be identical to that taken from in vitro studies due to factors such as temperature, tongue movement, humidity, and illumination. Moreover, differences in the manufacturer and the material of scan body could be another influencing factor in the accuracy of image matching. As such, future research that investigates a diverse range of intraoral scanners and scan bodies is required, followed by related in vivo research.

\section{CONCLUSION}

The improperly scanned data of a scan body can negatively affect the accuracy of virtual implant positioning in CAD software due to incorrect scan body image matching. Within the limitations of the present study, scan body images with a surface deficiency of less than $10 \%$ are recommended in order to reduce registration errors when determining the position of the implant.

\section{ORCID}

Se-Won Park bttps://orcid.org/0000-0003-2850-0333

Yong-Do Choi https://orcid.org/0000-0001-9098-9104

Du-Hyeong Lee https://orcid.org/0000-0003-2803-7457

\section{REFERENCES}

1. Christensen GJ. Will digital impressions eliminate the current problems with conventional impressions? J Am Dent Assoc 2008;139:761-3.

2. Di Fiore A, Vigolo P, Graiff L, Stellini E. Digital vs conventional workflow for screw-retained single-implant crowns: A comparison of key considerations. Int J Prosthodont 2018;31: 
$577-9$.

3. Haddadi Y, Bahrami G, Isidor F. Evaluation of operating time and patient perception using conventional impression taking and intraoral scanning for crown manufacture: A split-mouth, randomized clinical study. Int J Prosthodont 2018;31:55-9.

4. Winstanley RB, Carrotte PV, Johnson A. The quality of impressions for crowns and bridges received at commercial dental laboratories. Br Dent J 1997;183:209-13.

5. Christensen GJ. Impressions are changing: deciding on conventional, digital or digital plus in-office milling. J Am Dent Assoc 2009;140:1301-4.

6. Londono J, Abreu A, Baker PS, Furness AR. Fabrication of a definitive obturator from a 3D cast with a chairside digital scanner for a patient with severe gag reflex: a clinical report. J Prosthet Dent 2015;114:735-8.

7. Millstein PL. Determining the accuracy of gypsum casts made from type IV dental stone. J Oral Rehabil 1992;19:23943.

8. Kim M, Kim J, Mai HN, Kwon TY, Choi YD, Lee CH, Lee $\mathrm{DH}$. Comparative clinical study of the marginal discrepancy of fixed dental prosthesis fabricated by the milling-sintering method using a presintered alloy. J Adv Prosthodont 2019;11: 280-5.

9. Grünheid T, McCarthy SD, Larson BE. Clinical use of a direct chairside oral scanner: an assessment of accuracy, time, and patient acceptance. Am J Orthod Dentofacial Orthop 2014;146:673-82.

10. Flügge TV, Schlager S, Nelson K, Nahles S, Metzger MC. Precision of intraoral digital dental impressions with iTero and extraoral digitization with the iTero and a model scanner. Am J Orthod Dentofacial Orthop 2013;144:471-8.

11. Jeon JH, Hwang SS, Kim JH, Kim WC. Trueness and precision of scanning abutment impressions and stone models according to dental CAD/CAM evaluation standards. J Adv Prosthodont 2018;10:335-9.

12. Güth JF, Keul C, Stimmelmayr M, Beuer F, Edelhoff D. Accuracy of digital models obtained by direct and indirect data capturing. Clin Oral Investig 2013;17:1201-8.

13. Pan S, Guo D, Zhou Y, Jung RE, Hämmerle CHF, Mühlemann S. Time efficiency and quality of outcomes in a model-free digital workflow using digital impression immediately after implant placement: A double-blind self-controlled clinical trial. Clin Oral Implants Res 2019;30:617-26.

14. Alsharbaty MHM, Alikhasi M, Zarrati S, Shamshiri AR. A clinical comparative study of 3-dimensional accuracy between digital and conventional implant impression techniques. J Prosthodont 2019;28:e902-8.

15. Fluegge T, Att W, Metzger M, Nelson K. A novel method to evaluate precision of optical implant impressions with commercial scan bodies-An experimental approach. J Prosthodont 2017;26:34-41.

16. Lee SJ, Betensky RA, Gianneschi GE, Gallucci GO. Accuracy of digital versus conventional implant impressions. Clin Oral Implants Res 2015;26:715-9.

17. Keeling A, Wu J, Ferrari M. Confounding factors affecting the marginal quality of an intra-oral scan. J Dent 2017;59:33-40.

18. Kim JH, Kim KB, Kim SH, Kim WC, Kim HY, Kim JH.
Quantitative evaluation of common errors in digital impression obtained by using an LED blue light in-office CAD/ CAM system. Quintessence Int 2015;46:401-7.

19. Lee DH. Digital approach to assessing the 3-dimensional misfit of fixed dental prostheses. J Prosthet Dent 2016;116:8369.

20. Chen Y, Medioni G. Object modelling by registration of multiple range images. Image Vision Comput 1992;10:145-5.

21. Kim JE, Amelya A, Shin Y, Shim JS. Accuracy of intraoral digital impressions using an artificial landmark. J Prosthet Dent 2017;117:755-61.

22. Seo SW, Lee WS, Byun JY, Lee KB. A standardization model based on image recognition for performance evaluation of an oral scanner. J Adv Prosthodont 2017;9:409-15.

23. Andriessen FS, Rijkens DR, van der Meer WJ, Wismeijer DW. Applicability and accuracy of an intraoral scanner for scanning multiple implants in edentulous mandibles: a pilot study. J Prosthet Dent 2014;111:186-94.

24. Haddadi Y, Bahrami G, Isidor F. Effect of software version on the accuracy of an intraoral scanning device. Int J Prosthodont 2018;31:375-6.

25. Pesce P, Pera F, Setti P, Menini M. Precision and accuracy of a digital impression scanner in full-arch implant rehabilitation. Int J Prosthodont 2018;31:171-5.

26. Koch GK, Gallucci GO, Lee SJ. Accuracy in the digital workflow: From data acquisition to the digitally milled cast. J Prosthet Dent 2016;115:749-54.

27. Boeddinghaus M, Breloer ES, Rehmann P, Wöstmann B. Accuracy of single-tooth restorations based on intraoral digital and conventional impressions in patients. Clin Oral Investig 2015;19: 2027-34.

28. Kim RJ, Park JM, Shim JS. Accuracy of 9 intraoral scanners for complete-arch image acquisition: A qualitative and quantitative evaluation. J Prosthet Dent 2018;120:895-903.

29. Hayama H, Fueki K, Wadachi J, Wakabayashi N. Trueness and precision of digital impressions obtained using an intraoral scanner with different head size in the partially edentulous mandible. J Prosthodont Res 2018;62:347-52.

30. Medina-Sotomayor P, Pascual-Moscardó A, Camps I. Accuracy of four digital scanners according to scanning strategy in complete-arch impressions. PLoS One 2018;13:e0202916.

31. Müller P, Ender A, Joda T, Katsoulis J. Impact of digital intraoral scan strategies on the impression accuracy using the TRIOS Pod scanner. Quintessence Int 2016;47:343-9.

32. Giménez B, Özcan M, Martínez-Rus F, Pradíes G. Accuracy of a digital impression system based on active wavefront sampling technology for implants considering operator experience, implant angulation, and depth. Clin Implant Dent Relat Res 2015;17:e54-64. 